\title{
BOWEL PERFORATION DURING CATHETER REMOVAL AFTER THE SIXTH MONTH OF PERITONEAL DIALYSIS TERMINATION
}

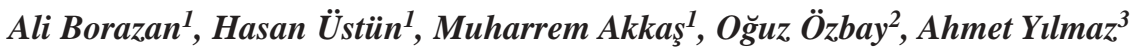 \\ Kocaeli University, Faculty of Medicine: Department of Internal medicine ${ }^{1}$, Department of General Surgery ${ }^{2}$, Department \\ of Internal Medicine and Nephrology ${ }^{3}$
}

Summary: Continuous ambulatory peritoneal dialysis (CAPD) is widely used in the treatment of end stage renal failure patients. Delayed injury to the bowel is an uncommon complication of CAPD catheter. In this article, we presented a case of bowel perforation during catheter removal after the sixth month of peritoneal dialysis termination.

Key words: Continuous ambulatory peritoneal dialysis; Catheter complication; Colon perforation

\section{Introduction}

Continuous ambulatory peritoneal dialysis (CAPD) is a safe and widely used mode of renal replacement therapy for patients with end-stage renal failure. It has a few complications such as peritonitis, catheter migration, haemoperitoneum, intestinal obstruction and intestinal erosion $(1,8,9)$. Organ laceration and/or perforation can be seen during insertion of the dialysis catheter, but delayed injury to the bowel is an uncommon complication.

\section{Case}

The patient, a 36-year-old male with end stage renal failure of unknown aetiology, commenced CAPD in June 1997 via Tenchkhoff catheter inserted through a transverse incision into the pelvis. Left hemithorax ampiyema was developed in the patient in July 2001 and medical treatment and closed drainage with chest tube were applied following fifteen days. Then the decortication procedure was performed because of persistent ampiyema and the patient was transferred to hemodialysis. The patient was confused about preferring which dialysis program was convenient for him, so that the catheter removal was delayed for 6 months. Then the patient was decided to continue on hemodialysis. In December 2001 the catheter foully smelled with faeces contamination of the last $3 \mathrm{~cm}$ portion. Drainage catheter was inserted through incision side up to Douglas pouch. Oral nutrition intake of the patient was stopped and commenced on intravenous tazocilline and metranidazol as antibacterial therapy. The bowel investigation procedures and surgical exploration were not performed because of incomplete bowel preparation. During hospitalization period the patient had no abdominal pain symptom and fever. The physical examination revealed no abdominal defense or rebound. No fluid discharge was seen within the drainage catheter inserted to the patient's Douglas pouch. All biochemical and complete blood count laboratory findings of the patient were in normal ranges during hospitalization period. Fifteen days later following bowel perforation, the patient started to take oral nutrition and performed barium enema and colonoscopy showed no pathological findings. The faecal sample at the tip of catheter was cultured and normal bowel flora was noted. Antibacterial treatment was stopped and the patient was discharged from the hospital. The patient is still on hemodialysis program with three sessions in a week.

\section{Discussion}

Perforation and injury to the intra-abdominal viscus are well-recognized complications of peritoneal dialysis catheter placement, and occurs in approximately $1.3-1.6 \%$ of all peritoneal dialysis placements $(1,8)$.

Generally CAPD terminates because of catheter dysfunction, frequent peritonitis attacks or many other causes (9). Wie didn't observe any peritonitis or other complications in the case during dialysis period and CAPD was terminated just because of left hemithorax ampiyema and performed decortication procedure.

Delayed bowel erosion and/or perforation due to CAPD catheter was reported in the literature $(1,5)$. In our case the catheter was removed in elective manner after 6 months of CAPD termination. We observed that catheter's end was contaminated with faeces. 
The treatment of bowel perforation due to peritoneal dialysis catheter is bed rest, antibacterial drugs and parenteral nutrition, or surgery $(1,6)$. In our case, bowel preparation could not be done so that surgery was postponed and conservative approach as antibiotics, parenteral nutrition and bed rest employed. This treatment was completed successfully.

Perforation of a viscus by a peritoneal catheter usually occurs in the context of dormant peritoneal dialysis. Brady et al. (2) reported an immunosuppressed renal transplant recipient case with bowel erosion due to a dormant peritoneal catheter. In intermittent peritoneal dialysis, the absence of dialysate fluid allows the catheter to irritate the peritoneum and bowel directly. It can occur also in ongoing CAPD

The late perforation of posterior peritoneum (4), urinary bladder (7) and rupture of splenic infarct (3) are described in the literature. The perforation localization in our case was suggested as distal to the transverse colon due to formed figure of faeces at the tip of catheter.

We conclude that bowel perforation might be seen even after existence of long interval between CAPD termination without any catheter related complication and catheter removal. The physician should be alert about the catheter induced organ laceration and/or perforation in all circumstances.

\section{References}

1. Balaji V, Digard N, Wise MH. Delayed bowel erosion due to functioning chronic ambulatory peritoneal dialysis catheter. Nephrol Dial Transplant 1996:11:368-9.

2. Brady HR, Abraham G, Oreopoulos DG, Cardella CJ. Bowel erosion due to a dormant peritoneal catheter in immunosuppressed renal transplant recipient. Perit Dial Int 1988;8:163.

3. Kanagasundaram NS, Macdougall IC, Turney JH. Massive haemoperitoneum due to rupture of splenic infarct during CAPD. Nephrol Dial Transplant 1998;13:2380-1.

4. Rodriguez-Perez JC, Palop L, Plaza C, Arrieta J. Perforation and/or laceration of posterior peritoneum in CAPD. Perit Dial Bull 1984;3:141.

5. Rotellar C, Sivarajan S, Mazzoni J et al. Bowel perforation in CAPD patients. Perit Dial Int 1992;12:396-8.

6 Thibodeaux LC. Bowel perforation associated with continuous ambulatory peritoneal dialysis. Nephron 1995;70:265.

7 Vargamezis V, Pasadakis P, Thodis E, Ethimiadou A, Maltezos E, Kotsiou S. Late perforation of bladder as a complication of an unused straight Tenckhoff catheter. Perit Dial Bull 1988;8:55-62.

8 Volpe della M, Iberti M, Ortensia A et al. Erosion of the sigmoid by a permanent peritoneal catheter. Perit Dial Bull 1984;4:108.

9 Watson LC, Thompson JC. Erosion of the colon by a long-dwelling peritoneal dialysis catheter. JAMA 1980;243:2156-7.

Submitted January 2003.

Accepted March 2003.

Hasan Üstün, M.D., Harpsundsvagen 9, 2tr, 12458 Bandhagen, Stockholm, Sweden. e-mail: ustunhtr@hotmail.com 